\title{
Destabilization of EpCAM dimer is associated with increased susceptibility towards cleavage by TACE
}

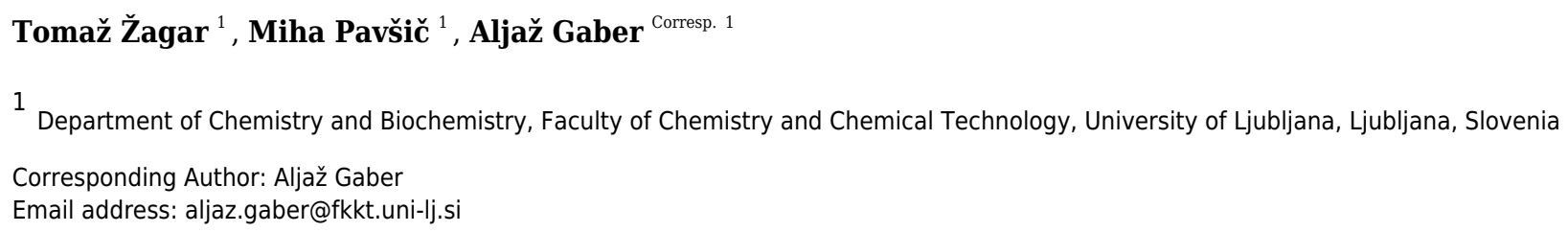

The cell-surface protein EpCAM is a carcinoma marker utilized in diagnostics and prognostics, and a promising therapeutic target. It is involved in nuclear signaling via regulated intramembrane proteolysis (RIP). Many aspects of this process are not fully understood, including the events at the molecular level leading to the exposure of cleavage sites, buried at the dimerization interface. To investigate the effect of dimer stability on cleavage susceptibility we prepared two mutants of human EpCAM ectodomain: a monomeric form, and a disulfide-stabilized dimeric form. We show that the disulfide-stabilized dimer is resistant to tumor necrosis factor- $\alpha$-converting enzyme (TACE) cleavage, while the monomeric form is more susceptible than the predominantly dimeric wild type. This provides experimental evidence that the oligomeric state of EpCAM is a determinant in RIP and demonstrates the usefulness of the oligomeric state-specific mutants in investigations of EpCAM biological function. 


\title{
1 Destabilization of EpCAM dimer is associated with
}

\section{2 increased susceptibility towards cleavage by TACE}

3

4

5

6

\author{
Tomaž Žagar ${ }^{1}$, Miha Pavšič ${ }^{1}$, Aljaž Gaber ${ }^{1}$
}

${ }^{1}$ Department of Chemistry and Biochemistry, Faculty of Chemistry and Chemical Technology, University of Ljubljana, Ljubljana SI 1000, Slovenia

Corresponding Author:

Aljaž Gaber

Večna pot 113, Ljubljana SI 1000, Slovenia

Email address: aljaz.gaber@fkkt.uni-lj.si

\section{Abstract}

The cell-surface protein EpCAM is a carcinoma marker utilized in diagnostics and prognostics, and a promising therapeutic target. It is involved in nuclear signaling via regulated intramembrane proteolysis (RIP). Many aspects of this process are not fully understood, including the events at the molecular level leading to the exposure of cleavage sites, buried at the dimerization interface. To investigate the effect of dimer stability on cleavage susceptibility we prepared two mutants of human EpCAM ectodomain: a monomeric form, and a disulfidestabilized dimeric form. We show that the disulfide-stabilized dimer is resistant to tumor necrosis factor- $\alpha$-converting enzyme (TACE) cleavage, while the monomeric form is more susceptible than the predominantly dimeric wild type. This provides experimental evidence that the oligomeric state of EpCAM is a determinant in RIP and demonstrates the usefulness of the oligomeric state-specific mutants in investigations of EpCAM biological function.

\section{Introduction}

Epithelial cell adhesion molecule (EpCAM, CD326) is a homodimeric type I cell-surface glycoprotein, frequently overexpressed in epithelial cancers (Went et al., 2004). EpCAM overexpression in carcinomas is linked to cancer proliferation, migration, and metastasis (Trzpis et al., 2007), and is associated with poor prognosis (Spizzo et al., 2002, 2004; Varga et al., 2004; Fong et al., 2007; Brunner et al., 2008; Scheunemann et al., 2008; Chen et al., 2014). This makes it a promising target for cancer diagnostics and treatment (reviewed in ref. (Simon et al., 2013)).

EpCAM exerts its biological function as a regulator of epithelial cell-cell adhesion (Gires et al., 2020) and is involved in signaling via two mechanisms - regulated intramembrane proteolysis (RIP) (Maetzel et al., 2009) and interaction with epithelial growth factor receptor (EGFR) (Pan et al., 2018; Liang et al., 2018). In RIP, EpCAM is first cleaved by $\alpha$-secretase ADAM10 or ADAM17, also known as tumor necrosis factor- $\alpha$-converting enzyme (TACE), within its extracellular part (EpEX), which leads to the release of the almost complete ectodomain, except for a short 2,4 $\mathrm{kDa}$ part that remains tethered to the membrane. Next, EpCAM is processed by 
42 the $\gamma$-secretase complex, resulting in a soluble short intracellular tail (EpIC). EpIC then interacts

43 with proteins of $\beta$-catenin/Wnt-signaling pathway to form the EpIC-FHL2-Lef1- $\beta$-catenin

44 complex (Maetzel et al., 2009; Tsaktanis et al., 2015), which in turn regulates expression of

45 oncogenes (Maetzel et al., 2009; Lu et al., 2010; Huang et al., 2011; Chaves-Pérez et al., 2013;

46 Yu, Ma \& Wang, 2017).

47

48 The quaternary structure of EpCAM appears to be inherently linked to its biological function.

49 Initial models predicted various oligomeric states; however, later it has been demonstrated that

50 the ectodomain forms a stable dimer (Pavšič et al., 2014; Gaber et al., 2018). This dimer is also

51 representative of the full-length molecule where the transmembrane helix dimerization could

52 further contribute to the dimer stability (Pavšič et al., 2014; Gaber et al., 2018). Mapping of the

53 TACE cleavage sites onto the EpEX dimerization surface (Tsaktanis et al., 2015) indicated that

54 the cleavage by TACE can take place only when EpCAM is in a monomeric state. Molecular

55 docking of the TACE catalytic domain on EpEX (Gaber, Lenarčič \& Pavšič, 2020) also supports

56 this hypothesis by demonstrating that EpCAM dimerization sterically hinders access of TACE to

57 the cleavage site. However, the effect of EpCAM oligomeric state on susceptibility to TACE

58 cleavage has never been experimentally addressed, partly due to the inability to manipulate the

$59 \mathrm{EpCAM} / \mathrm{EpEX}$ oligomeric state.

60

61 Here, we present two designed EpEX mutants with distinct oligomeric states. First, by analysis

62 of EpEX dimerization surface as found in crystal structure and further explored by molecular

63 dynamics simulations, we devised a set of three single-site mutations that prevent EpEX

64 dimerization and therefore result in persistently monomeric EpEX. Second, we generated a

65 covalently stabilized EpEX dimer by introducing a cysteine residue at the C-terminal tail of

66 EpEX, leading to the formation of a disulfide bridge between the two subunits. Third, we

67 demonstrate that these two mutant proteins can be employed to study the effect of EpEX's

68 oligomeric state on its interactions with other proteins. By comparing the extent of their

69 proteolytic degradation with TACE's ectodomain (TACE-EX) in a solution we provide

70 experimental evidence that EpCAM can only be efficiently cleaved as a monomer, strongly

71 indicating the importance of the role of EpCAM's oligomerization in the regulation of RIP. 


\section{Materials \& Methods}

75

76

77

78

79

80

81

82

83

84

85

86

87

88

89

90

91

92

93

94

95

96

97

98

99

100

101

102

103

104

105

106

107

108

109

110

111

\section{In silico construction and Molecular dynamics simulations of EpEX}

As input structures EpEX dimer from the crystal structure (PDB ID 4mzv) (Pavšič et al., 2014) and its in silico mutated TYloop ${ }^{\text {mut }}$ form (K83D, P84D, L88D) were used. The mutations were introduced using UCSF Chimera (Pettersen et al., 2004). First, topology files were prepared using VMD 1.9.3 with the psfgen plugin (http://www.ks.uiuc.edu/Research/vmd/) (Humphrey, Dalke \& Schulten, 1996). Briefly, protein molecules were solvated (20 ̊ margin on each side, giving a box of approximately $100 \times 100 \times 100 \AA$ ) and the system neutralized by the introduction of sodium or chloride ions. Next, all-atom molecular dynamics runs were performed using NAMD (http://www.ks.uiuc.edu/Research/namd/) 2.11 (Phillips et al., 2005) under periodic boundary conditions and using full-system periodic electrostatics. To prevent the introduction of artefacts, the protein atoms were kept at a fixed position during the initial minimization step, and only the water molecules and ions were allowed to move freely. Following this, restrictions were removed and the system was equilibrated at a constant pressure of $1 \mathrm{~atm}$ using Langevin piston, and a constant temperature of $310 \mathrm{~K}$ using Langevin dynamics for $5 \mathrm{~ns}$ with a timestep of $2 \mathrm{fs}$. Trajectory files were analyzed using UCSF Chimera (Pettersen et al., 2004) to obtain inter-residue distance $v s$. time. To obtain the inter-residue contact network we used USCF Chimera in conjunction with Cytoscape 3.8.2 (Shannon et al., 2003) and StructureViz2 plugin (Morris et al., 2007).

\section{Protein cloning, expression, and purification}

Both EpEX mutants-TYloop ${ }^{\text {mut }}$ (K83D, P84D and L88D) and L264C - were derived from EpEX (residues 24-265) with mutated N-glycosylation sites (N74Q, N111Q and N198Q) (Pavšič et al., 2014). The final K265 is followed by residues LE, translated from XhoI scar, and a His6tag. The wild-type signal sequence was replaced with the honeybee signal sequence (MKFLVNVALVFMVVYISYIYA) to increase expression yields (Pavšič et al., 2014) in insect cells. Protein coding sequences were cloned in $\mathrm{pFastBac1}$. Recombinant bacmids were prepared in DH10MultiBac (Berger, Fitzgerald \& Richmond, 2004) according to the Bac-to-Bac system (Invitrogen) and transfected into insect cells Spodoptera frugiperda Sf9 (Thermo Fisher Scientific, USA) using Turbofect according to manufacturer's instructions (Thermo Fisher Scientific, USA). Baculoviral stock solution (V0) was amplified twice, each time for three days, to ensure high viral titer for efficient expression of recombinant proteins.

All proteins were expressed in insect cell line Spodoptera frugiperda Sf9 (Thermo Fisher Scientific, USA) and purified as described before (Pavšič et al., 2014). Harvesting was done three days post-infection with $\mathrm{V} 2$ viral stock, and the cell culture supernatant was obtained by centrifugation $\left(10 \mathrm{~min}\right.$ at $10,000 \times \mathrm{g}$ at $\left.4{ }^{\circ} \mathrm{C}\right)$. The $\mathrm{pH}$ of the cleared supernatant was adjusted to 8.0 by adding Tris- $\mathrm{HCl}$ to a final concentration of $2.5 \mathrm{mM}$ during mixing, and protease inhibitor 
112 phenylmethylsulfonyl fluoride (PMSF) was added to a final concentration of $1 \mathrm{mM}$. The solution

113 was centrifuged for the second time $\left(10 \mathrm{~min}\right.$ at $10,000 \times \mathrm{g}$ at $\left.4{ }^{\circ} \mathrm{C}\right)$ and applied to a $5 \mathrm{ml}$

114 cOmplete His-Tag Purification Column (Roche, Switzerland), previously equilibrated with 20

$115 \mathrm{mM}$ sodium phosphate buffer, $\mathrm{pH} 7.5,500 \mathrm{mM} \mathrm{NaCl}$. Recombinant proteins were eluted using

116 an imidazole gradient (final concentration of $500 \mathrm{mM}$ ) and dialyzed overnight against $20 \mathrm{mM}$

117 HEPES, $\mathrm{pH} 8$ at $4{ }^{\circ} \mathrm{C}$. The purification then proceeded with an ion-exchange chromatography on

$1185 \mathrm{ml}$ HiTrap Q HP (GE Healthcare, USA), equilibrated with $20 \mathrm{mM}$ HEPES, pH 8. Proteins were

119 eluted using $\mathrm{NaCl}$ gradient (final concentration of $500 \mathrm{mM}$ ). Fractions containing recombinant

120 proteins of interest (as determined by SDS-PAGE) were pooled and applied to size exclusion

121 column HiLoad ${ }^{\mathrm{TM}}$ 16/600 (GE Healthcare, USA), equilibrated with 20 mM HEPES, pH 8, 100

$122 \mathrm{mM} \mathrm{NaCl})$. Pooled fractions were concentrated and stored at $-80^{\circ} \mathrm{C}$.

123

124

125

126

127

128

129

Recombinant baculoviruses harboring fragment coding for TACE-EX (residues 1-824, with Nterminal honeybee melittin signaling sequence and C-terminal His6-tag) were prepared in the same way as the EpEX mutants. Also, the expression and purification procedure were the same, except for the addition of Halt ${ }^{\mathrm{TM}}$ Protease and Phosphatase Inhibitor Single-Use Cocktail, EDTA-Free (Thermo Fisher Scientific, USA) after the dialysis step.

\section{Molecular weight analysis}

132

Molecular weights (MWs) of the proteins were assessed by Size exclusion chromatography

133 coupled with right- and low-angle laser light scattering detectors (SEC-RALLS/LALLS system OMNISEC RESOLVE+REVEAL (Malvern Panalytical, UK) using a Superdex ${ }^{\circledR} 200$ Increase 10/300 GL column (GE Healthcare/Cytiva, USA) for protein separation. $150 \mu \mathrm{g}$ of each protein was injected per run and each protein sample was analyzed in a duplicate. The system operated at $25^{\circ} \mathrm{C}$ and a flow rate of $0.5 \mathrm{ml} / \mathrm{min}$. For all samples, the same buffer was used namely, $20 \mathrm{mM}$ HEPES, pH 7.5, $100 \mathrm{mM} \mathrm{NaCl}$. BSA (Thermo Fischer Scientific, USA) was used as a calibration standard. Retention volumes were adjusted according to BSA peak position. The MW distributions were calculated using the software OMNISEC version 11.20 (Malvern Panalytical, UK).

\section{CD analysis}

143 Circular dichroism spectra were recorded on a J-1500 CD spectrometer (Jasco, USA) in a $1 \mathrm{~mm}$ 144 quartz cuvette (Hellma, Germany) at $25^{\circ} \mathrm{C}$. Spectra were registered in the wavelength range 145 between 200 and $250 \mathrm{~nm}$ with scanning speed set to $20 \mathrm{~nm} / \mathrm{min}$ and the bandwidth and data pitch 146 to $1 \mathrm{~nm}$. Four scans per measured sample were averaged and corrected for the sample buffer (20

149 Proteolytic cleavage of EpEX and its mutants

150 Proteins were dialyzed against the reaction buffer $20 \mathrm{mM}$ sodium phosphate, $\mathrm{pH} 7.4,10 \mu \mathrm{M}$

$151 \mathrm{ZnCl}_{2}$, complemented with cOmplete ${ }^{\mathrm{TM}}$ Mini EDTA-free Protease Inhibitor Cocktail (Roche, 
152 Switzerland), using a Slide-A-Lyzer® MINI Dialysis device (10K MWCO membrane, 10-100

153

154

155

156

157

158

159

160

161

162

163

164

165

166

167

168

169

170

171

172

173

174

175

176

177

178

179

180

181

182

183

184

185

186

187

188

189

190

191

192

193

ul) (Thermo Fischer Scientific), according to manufacturer's instruction.

For a single cleavage experiment, $15 \mu \mathrm{g}$ of EpEX or its mutant were mixed with $7.5 \mu \mathrm{g}$ of

TACE-EX in a final volume of $30 \mu \mathrm{l}$ and incubated for 0,2 , or $6 \mathrm{~h}$ at $37^{\circ} \mathrm{C}$ with mixing at 300

RPM. The reaction was stopped by the addition of $4 \times$ SDS loading buffer containing DTT, and subsequent incubation at $95^{\circ} \mathrm{C}$ for $5 \mathrm{~min}$. The control samples without the TACE-EX were incubated for $6 \mathrm{~h}$, while the initial samples were not incubated at $37^{\circ} \mathrm{C}$.

For the Western blot analysis, one-tenth of the reaction sample was separated using SDS-PAGE. Separated proteins were transferred onto Immobilon®-FL PVDF (Merck Millipore, USA) membrane using wet transfer system Mini Trans-Blot ${ }^{\circledR}$ (Bio-Rad, USA). Blocking was done using fluorescent blocking solution Immobilon ${ }^{\circledR}$ Block - FL (Merck Millipore, USA) supplemented with $0.2 \%$ Tween 20 for 1 hour at room temperature during constant mixing. Mouse anti-His Tag antibodies (ABIN195462, Antibodies-Online, Germany) were diluted 1:1000 directly into the blocking solution and the membrane was incubated for an additional hour. After three 5-minute washes with TBST (0.2\% Tween 20), the membrane was incubated in goat anti-mouse IgG $(\mathrm{H}+\mathrm{L})$ superclonal recombinant secondary antibodies conjugated with Alexa Fluor 647 dye (Thermo Fischer Scientific, USA) diluted 1:5000 in blocking solution supplemented with $0.2 \%$ Tween 20 at room temperature for $1 \mathrm{~h}$ and washed six times for five minutes with TBST. Detection was performed using ChemiDoc ${ }^{\mathrm{TM}}$ MP imaging system (Bio-Rad, USA) with a preset program for imaging AlexaFluor 647 dye. Quantification was done using software ImageLab version 6.0.1 (Bio-Rad, USA).

The band intensities of three different blots were first scaled according to the sum of the signal of the initial samples. For each protein, the average of scaled control samples incubated without the TACE-EX was used for normalization.

\section{Statistics}

Results of proteolytic cleavage experiments are represented as a mean value \pm SEM. One-way ANOVA $(\mathrm{P}<0.001)$. Analyses were done with GraphPad Prism version 8.

\section{Results}

\section{Rational design of monomeric and dimeric mutants}

We devised mutations that either additionally stabilize or destabilize EpEX dimer. For this, the crystal structure of EpCAM ectodomain dimer was used as the representation of the native state (Pavšič et al., 2014). In this dimer, the long loop of the thyroglobulin domain (TYloop) forms extensive interactions with the $\beta$-sheet in the C-terminal domain (CD) of the juxtaposed subunit. Here, electrostatic interactions between Arg80, Arg81, and Lys83 (all within the TYloop), and a negatively charged patch formed by Glu147, Glu187, Asp194 (all within $\beta$-sheet) appear to be critical for dimer stabilization (Pavšič et al., 2014) (Fig. 1A). In the design process three

PeerJ reviewing PDF | (2021:03:58778:1:1:NEW 22 Apr 2021) 
194 additional aspects were considered: (1) mutations within TYloop, which is in monomeric species

195

196

197

198

199

200

201

202

203

204

205

206

207

208

209

210

211

212

213

214

215

216

217

218

219

220

221

222

223

224

225

226

227

228

229

230

231

232

233

234

235

236

237

238

239 completely exposed to solvent and probably structurally disordered, most probably wouldn't interfere with subunit folding; (2) mutations introduced within the $\beta$-sheet of the C-terminal domain could interfere with domain folding; (3) protease-sensitive site Gly79-Arg80-Arg81 (Thampoe, Ng \& Lloyd, 1988; Perez \& Walker, 1989; Schön et al., 1993) has to be retained so that the mutant protein can potentially be used in experiments addressing processing at this site.

The mutant with impaired dimerization propensity was designed by introducing negatively charged residues into the TYloop, which would result in electrostatic repulsion between this region and the negatively charged patch within the $\beta$-sheet of the juxtaposed subunit. We prepared in silico mutants where residues in the range 83-88 were changed one-by-one to aspartates using UCSF Chimera (Pettersen et al., 2004; Goddard, Huang \& Ferrin, 2007); for each mutation, the most probable rotamer from the Dunbar library was chosen (Shapovalov \& Dunbrack, 2011). The effect of mutations on dimer stability was evaluated using the 'Protein interfaces, surfaces and assemblies' service PISA at the European Bioinformatics Institute (Krissinel \& Henrick, 2007). The highest impact on solvation free energy gain upon the formation of the dimer interaction interface was achieved by a set of three mutations K83D, P84D and L88D, and this mutant was named TYloop ${ }^{\text {mut }}$ (Fig. 1A, Table S1).

To get detailed insight into the dimer-stabilizing interactions and their time distribution we performed an all-atom $5 \mathrm{~ns}$ molecular dynamics (MD) simulation and analyzed the inter-residue contacts between the two subunits of the native-like EpEX dimer in terms of their frequency (Fig. 1B). We observed several frequent ionic interactions (salt bridges) as already inferred from the static crystal structure; however, the MD simulation revealed some additional prominent residue clusters, particularly those with Leu88 in the center, and a small cluster involving Pro84. This is in good agreement with the dimer interface analysis described above. To evaluate the effect of the designed mutations in silico, we performed an analogous MD simulation run also with the mutated EpEX form - the EpEX TYloop ${ }^{\text {mut }}$. Trajectory analysis of inter-residue distance vs. time for selected residue pairs (K83-E147, P84-W143, L88-Y251 for wild type, and D83E147, D84-W143, D88 - Y251 for the EpEX TYloop ${ }^{\text {mut }}$ ) revealed that the distance $v s$. time is very stable for the wild type form (Fig. 1C). On the contrary, the distance plot for the equivalent atom pairs shows a considerable jump to higher values indicating the start of dimer dissociation (Fig. 1D), except for the residue pair involving Y251 where the distance in the wild type form is already larger. This further supports the proposed destabilizing effect of designed mutations of the EpEX TYloop ${ }^{\text {mut }}$ form.

A covalently stabilized dimer mutant was prepared by introducing a cysteine residue (L264C) near the C-terminus of the EpEX (Fig. 1A). The formation of an EpEX dimer via native interactions would bring the two cysteine residues from juxtaposed subunits into close proximity thus enabling the formation of a disulfide bond, thereby covalently linking the subunits and preventing their dissociation. The oxidative potential of the environment where the mutated site is located in the expressed protein - the lumen of the secretory pathway compartments or the extracellular space - would contribute to the probability that a stable disulfide bond is indeed formed. The C-terminus of EpEX was shown to be flexible and the mutation spot is distant from the major well-structured part of the subunit; therefore, the mutation L264C was not predicted to introduce significant structural perturbations (Gaber et al., 2018). 
A mutant non-glycosylated EpEX variant was used as starting template for both described mutants to avoid the effect of heterogeneous and non-native glycosylation due to expression in insect cells. It had already been demonstrated that this variant forms stable dimers (Pavšič et al., 2014; Gaber et al., 2018).

The designed EpEX mutants occupy distinct oligomeric states

EpEX with native-like dimerization propensity and both designed mutant EpEX variants (TYloop ${ }^{\text {mut }}$ and L264C) were expressed in insect cells and purified using a series of chromatographic runs. Final yields of designed mutants were lower than that of EpEX - approx. $2 \mathrm{mg}$ of purified protein per liter of cell culture, compared to 4-5 mg for EpEX; however, they

251

252 were sufficient for further analysis. The CD spectra of all three purified proteins are very similar (Fig. 2A) which indicates that the introduced mutations did not significantly affect the EpEX structure and that both TYloop ${ }^{\text {mut }}$ and L264C are properly folded.

We investigated the oligomeric state of EpEX, TYloop ${ }^{\text {mut }}$ and L264C using size exclusion

256

257

258

259

260

261

262

263

264

265

266 chromatography (SEC), MW determination via light scattering, and SDS-PAGE. First, in SEC elution chromatograms all variants gave a single peak (Fig. 2B). This indicates that there are no significant aggregates in any of the samples and that there is one predominant oligomeric species present in each of the samples. The peaks relative to the TYloop ${ }^{\text {mut }}$ and L264C are symmetric, while the EpEX peak exhibits slight tailing which could be attributed to the small amount of monomeric species. The position of the peak for EpEX and L264C overlap, suggesting that their oligomeric state is the same - a dimer, which has already been demonstrated for EpEX (Pavšič \& Lenarčič, 2011; Pavšič et al., 2014; Gaber et al., 2018).TYloop ${ }^{\text {mut }}$ elutes at higher elution volume than EpEX and L264C indicating a smaller hydrodynamic radius, indicating a different oligomeric state than for EpEX and L264C.

Next, we used SEC-RALLS/LALLS to determine the absolute MW of the species present in the solution (Fig. 2C). The determined MW of the species present in the main part of the EpEX peak is $52.8 \pm 0.4 \mathrm{kDa}$, while for the tailing part it decreases to $30 \mathrm{kDa}$. These two extremes roughly correspond to the dimer and monomer with calculated MW of 54.4 and $28.2 \mathrm{kDa}$, respectively, confirming the equilibrium between the two species. On the other hand, the plot of MW vs. elution volume for the two mutants indicates that each mutant is present in the solution as a species with a single oligomeric state. The determined MW for TYloop ${ }^{\text {mut }}$ of $28.7 \pm 0.1 \mathrm{kDa}$ is in good agreement with the monomeric form, while the MW for L264C (56.0 $\pm 0.1 \mathrm{kDa})$ indicates a dimeric form. The latter, together with no observed peak tailing, also confirms the expectation that the disulfide bond formed between the introduced cysteine residue from juxtaposed dimer subunits would prevent the dimer dissociation.

280

Last, we used SDS-PAGE under reducing and non-reducing conditions to analyze whether the disulfide linkage between the subunits of the L264C dimer indeed formed as designed (Fig. 2D). Under reducing conditions, EpEX and both mutants have the same electrophoretic mobility, and the apparent MW corresponds to that calculated from the amino acid sequence $(28 \mathrm{kDa})$.

283

284 However, under non-reducing conditions, the electrophoretic mobility of the L264C mutant is significantly lower and corresponds to a dimer $(56 \mathrm{kDa})$, in contrast to the EpEX and TYloop ${ }^{\text {mut }}$ 
285

286

287

288

289

290

291

292

293

294

295

296

297

298

299

300

301

302

303

304

305

306

307

308

309

310

311

312

313

314

315

316

317

318

319

320

321

322

323

324

325

326

327

328

329

where electrophoretic mobility is unchanged. Only a very small amount of L264C mutant is monomeric under reducing conditions, which clearly shows that the majority of subunits in L264C are covalently linked together in pairs by a disulfide bond that results from the L264C mutation.

Based on SEC elution profiles, determined MW and SDS-PAGE analysis we conclude that we have successfully prepared two EpEX mutants with distinct oligomeric states. As neither of them exhibits equilibrium between the two monomeric and dimeric states, they are suitable candidates for further investigations of how the oligomeric state of EpEX affects EpCAM's role in cellular processes such as RIP-mediated signaling.

\section{EpEX monomeric mutant is more prone to degradation by TACE}

Mapping of TACE cleavage sites to EpEX dimer (Tsaktanis et al., 2015) indicated that the oligomeric state of EpCAM could play a role in the regulation of RIP. Namely, the cleavage sites appear to be accessible only if the EpCAM is monomeric. To address this experimentally we investigated the susceptibility of EpEX, plus the TYloop ${ }^{\text {mut }}$ (monomer) and L264C (dimer) mutants towards cleavage by TACE ectodomain (TACE-EX) in vitro.

The cleavage extent was estimated via quantification of the protein remaining uncleaved, which was detected using anti-His6-tag antibodies targeting the His6-tag present in all three EpEX variants. As the tag gets released after the cleavage, the extent of cleavage can be evaluated by measuring the reduction of the signal (Fig. 3A). This approach was selected because the cleavage site by TACE is relatively close to the $\mathrm{C}$-terminus of EpEX, and the cleavage results in the liberation of a short fragment $(2.1 \mathrm{kDa})$ which is challenging to be detected and accurately quantified using SDS-PAGE. At the same time, the difference in MW between non-cleaved and cleaved is EpEX too small; therefore, the cleavage product is not easily resolvable from the uncleaved EpEX.

Our results show that roughly a quarter of the EpEX was cleaved after $2 \mathrm{~h}$, and almost $40 \%$ after $6 \mathrm{~h}$ (Fig. 3B). In contrast to this, only insignificant cleavage of L264C was observed (Fig. 3B, C). This confirms the hypothesis that cleavage by TACE-EX is inefficient when EpCAM is a dimer. On the other hand, the cleavage of TYloop ${ }^{\text {mut }}$ is significantly more efficient compared to EpEX at both time points, as almost more than $75 \%$ was processed after $2 \mathrm{~h}$ and more than $90 \%$ after $6 \mathrm{~h}$, further confirming that the oligomeric state is implicated in the regulation of RIP. At the same time, this demonstrates that our designed mutants are indeed useful to study the role of EpCAM oligomerization in RIP. Parallel SDS-PAGE analysis (Fig. 3D) of the same samples that were analyzed by Western blotting shows that the band intensity corresponding to all three EpEX variants is the same at each time point which indicates that the His6-tag signal of cleaved protein indeed diminishes due to cleavage near the $\mathrm{C}$-terminus.

Surprisingly, the signal corresponding to TACE-EX on our Western blots could only be observed after a prolonged exposition. Since the TACE-EX bears a C-terminal His6-tag-similarly to the EpEX variants - a stronger signal would be expected on a Western blot after detection using anti-His6-antibody (Fig. 3A). However, on SDS-PAGE the band corresponding to TACE-EX is visible (Fig. 3D). The apparent MW is larger than that calculated from the amino acid sequence 
$330(52 \mathrm{kDa})$, and the difference could be attributed to glycosylation at one or more of the nine $\mathrm{N}$ -

331 glycosylation sites present in TACE-EX. We speculate that the reduced signal intensity on

332 Western blots is a result of a C-terminal truncation, which occurred during the purification,

333 resulting in the loss of His6-tag. Similar observations of the loss of C-terminal tags after

334 purifications were also observed by others for both TACE (Johannes, Bercher \& Blobel, 2000)

335

336

337

338

339

340

341

342

343

344

345

346

347

348

349

350

351

352

353

354

355

356

357

358

359

360

361

362

363

364

365

366

367

368

369

370

371

372

373

374 and its homologue ADAM10(Brummer et al., 2018). They were attributed to C-terminal autoproteolytic processing(Johannes, Bercher \& Blobel, 2000; Brummer et al., 2018).

We believe that the observed cleavage extent is indeed linked to the EpEX oligomeric state and activity of TACE-EX. First, all cleavage reaction mixtures contained a cocktail of inhibitors targeting proteases of all other classes. Next, all proteins were isolated using the same procedure and would thus have the same contaminants. However, when these proteins are incubated without TACE-EX (Fig. 3A, D), no degradation is observed.

\section{Discussion}

Homo-oligomerization has always been recognized as an important aspect of EpCAM function. Initial observations of its role in cell-cell adhesion were attributed to its ability to form cell-cell contacts through the self-assembly of EpCAM molecules from adjacent cells (Balzar et al., 2001; Trebak et al., 2001). A recent investigation of EpCAM oligomerization did not find any direct evidence of higher-order oligomerization, and it also confirmed speculations that EpCAM exists predominately as a cis-dimer - a dimer formed by the association of two subunits from the same cell (Gaber et al., 2018). However, differences between monomeric and dimeric forms of EpCAM and their specific functional roles were never investigated. This was largely due to the lack of options for confining EpCAM to either a monomeric or dimeric state without considerably changing experimental conditions, for example by lowering the $\mathrm{pH}$ (Pavšič \& Lenarčič, 2011). To make such investigations possible, we prepared both monomeric as well as an obligate-dimeric mutant of EpCAM soluble ectodomain.

We showed that three mutations in EpEX TY-loop (K83D, P84D and L88D) successfully prevent dimerization. This confirms conclusions based on EpEX crystal structure (Pavšič et al., 2014) that interactions between TY-loop and $\beta$-sheet are essential for dimer formation. Even at a concentration of $1.5 \mathrm{mg} / \mathrm{ml}(\sim 50 \mu \mathrm{M})$, the mutant TYloop ${ }^{\text {mut }}$ showed no signs of dimerization (Fig. 2B, C). The wild type-like EpEX is almost exclusively dimeric at this concentration (Fig. $2 \mathrm{~B}, \mathrm{C})$, which is also in agreement with previously published small-angle $\mathrm{x}$-ray scattering (SAXS) data, collected at this concentration (Gaber et al., 2018). While the wild type-like EpEX exists both as monomer and dimer (in equilibrium), the L264C mutant is constitutively dimeric (Fig. 2B, C), due to disulfide covalent linkage between the two subunits (Fig. 2D).

Using the prepared mutants, we also experimentally in vitro confirmed the role of EpCAM oligomeric state in RIP, specifically the first cleavage by TACE, for the first time. The lack of any significant TACE-EX cleavage of L264C mutant and the almost complete cleavage of TYloop ${ }^{\text {mut }}$ mutant (Fig.3) clearly show that EpCAM needs to be monomeric for the cleavage to take place. The wild-type-like EpEX, which was shown to be a stable dimer in solution, was also cleaved, although to a lesser extent than the monomeric mutant. We hypothesize that TACE-EX readily cleaves monomers as soon as they become available due to the dissociation of subunits in

375 a dimer. However, to evaluate the potential TACE innate ability to promote EpEX dissociation, 
376 experiments in cellula are needed. For example, an amphipathic $\alpha$-helical region at the C-

377 terminal part of TACE-EX called Conserved Adam seventeeN Dynamic Interaction Sequence

378 (CANDIS) was shown to be essential for substrate recognition in cleavage of type I

379 transmembrane proteins such as IL-R6 (Düsterhöft et al., 2014). Whether interaction with

380 CANDIS is also important for EpCAM cleavage and potentially even its oligomeric state is still

381 to be discovered. Since CANDIS also interacts with the cell membrane (Düsterhöft et al., 2015)

382 such experiments require the use of full-length membrane-embedded proteins to adequately

383 represent the native conditions.

384

385

386

387

388

389

390

391

392

393

394

395

396

397

398

399

400

401

402

403

404

405

406

407

408

409

410

\section{Acknowledgements}

412 We thank Prof. Brigita Lenarčič (University of Ljubljana, Faculty of Chemistry and Chemical

413

414

415

416

417

418

419

Although both mutant proteins have distinct oligomeric states in solution and constitute a useful system for functional studies of EpEX in vitro, their effect in full-length EpCAM remains to be evaluated before the conclusions are applied to EpCAM in a cellular context. Here, the EpCAM transmembrane $\alpha$-helix is predicted to play a role in dimer stabilization via their intramembrane dimerization (Pavšič et al., 2014). Therefore, the full-length protein with TYloop ${ }^{\text {mut }}$ mutations could thus still exist as a dimer, associated via the transmembrane regions. Even if this is indeed the case, we speculate that the abolished interactions between EpEXs would still lead to a less stable dimer that should be more prone to dissociation and consequently to proteolytic processing. On the other hand, it is unlikely EpCAM would get cleaved if subunits remain connected via the transmembrane $\alpha$-helixes, even if the interactions between the ectodomains are abrogated. The interaction between transmembrane regions should confine the movement of EpEX similarly to the disulfide bond in L264C, which is located at its C-terminus (Fig. 4).

\section{Conclusions}

To conclude, we have designed, purified and characterized oligomer-state specific mutants of EpCAM's ectodomain. With them, we have shown that the destabilization of EpCAM dimer increases its susceptibility towards cleavage by TACE and that the cleavage of a dimeric EpCAM is negligible. We believe that these mutants of EpCAM ectodomain will provide a valuable tool for elucidating the role of the EpCAM oligomeric state either in solution or on the cell surface. Illuminating the role of the individual oligomeric state in interaction with other proteins and how oligomerization itself is regulated will importantly contribute to the understanding of the underlying mechanisms of the biological function of EpCAM.

Technology) for helpful discussion on experiment design, data analysis and manuscript preparation. We thank Carol Brandi and Debbie Gill for proofreading the final version and for their critical grammatical corrections. NAMD was developed by the Theoretical and Computational Biophysics Group in the Beckman Institute for Advanced Science and Technology at the University of Illinois at Urbana-Champaign.

Peer] reviewing PDF | (2021:03:58778:1:1:NEW 22 Apr 2021) 


\section{References}

423

424

425

426

427

428

429

430

431

432

433

434

435

436

437

438

439

440

441

442

443

444

445

446

447

448

449

450

451

452

453

454

455

456

457

458

459

460

461

462

463

464

465

Balzar M, Briaire-de-Bruijn IH, Rees-Bakker HAM, Prins FA, Helfrich W, de Leij L, Riethmüller G, Alberti S, Warnaar SO, Fleuren GJ, Litvinov. 2001. Epidermal growth factor-like repeats mediate lateral and reciprocal interactions of Ep-CAM molecules in homophilic adhesions. Molecular and Cellular biology 21:2570-2580. DOI: 10.1128/MCB.21.7.2570.

Berger I, Fitzgerald DJ, Richmond TJ. 2004. Baculovirus expression system for heterologous multiprotein complexes. Nature biotechnology 22:1583-7. DOI: 10.1038/nbt1036.

Brummer T, Pigoni M, Rossello A, Wang H, Noy PJ, Tomlinson MG, Blobel CP, Lichtenthaler SF. 2018. The metalloprotease ADAM10 (a disintegrin and metalloprotease 10) undergoes rapid, postlysis autocatalytic degradation. The FASEB Journal 32:3560-3573. DOI: 10.1096/fj.201700823RR.

Brunner A, Schaefer G, Veits L, Brunner B, Prelog M, Ensinger C. 2008. EpCAM overexpression is associated with high-grade urothelial carcinoma in the renal pelvis. Anticancer research 28:125-8.

Chaves-Pérez A, MacK B, Maetzel D, Kremling H, Eggert C, Harréus U, Gires O. 2013. EpCAM regulates cell cycle progression via control of cyclin D1 expression. Oncogene 32:641-650. DOI: 10.1038/onc.2012.75.

Chen X, Ma W-Y, Xu S-C, Liang Y, Fu Y-B, Pang B, Xin T, Fan H-T, Zhang R, Luo J-G, Kang W-Q, Wang M, Pang Q. 2014. The overexpression of Epithelial cell adhesion molecule (EpCAM) in glioma. Journal of neuro-oncology. DOI: 10.1007/s11060-014-1459-5.

Düsterhöft S, Höbel K, Oldefest M, Lokau J, Waetzig GH, Chalaris A, Garbers C, Scheller J, Rose-John S, Lorenzen I, Grötzinger J. 2014. A Disintegrin and Metalloprotease 17 Dynamic Interaction Sequence, the Sweet Tooth for the Human Interleukin 6 Receptor. Journal of Biological Chemistry 289:16336-16348. DOI: 10.1074/jbc.M114.557322.

Düsterhöft S, Michalek M, Kordowski F, Oldefest M, Sommer A, Röseler J, Reiss K, Grötzinger J, Lorenzen I. 2015. Extracellular Juxtamembrane Segment of ADAM17 Interacts with Membranes and Is Essential for Its Shedding Activity. Biochemistry 54:5791-5801. DOI: 10.1021/acs.biochem.5b00497.

Fong D, Steurer M, Obrist P, Barbieri V, Margreiter R, Amberger A, Laimer K, Gastl G, Tzankov A, Spizzo G. 2007. Ep-CAM expression in pancreatic and ampullary carcinomas: frequency and prognostic relevance. Journal of Clinical Pathology 61:31-35. DOI: 10.1136/jcp.2006.037333.

Gaber A, Kim SJ, Kaake RM, Benčina M, Krogan N, Šali A, Pavšič M, Lenarčič B. 2018. EpCAM homo-oligomerization is not the basis for its role in cell-cell adhesion. Scientific Reports 8:13269. DOI: 10.1038/s41598-018-31482-7.

Gaber A, Lenarčič B, Pavšič M. 2020. Current View on EpCAM Structural Biology. Cells 9:1361. DOI: 10.3390/cells9061361.

Gires O, Pan M, Schinke H, Canis M, Baeuerle PA. 2020. Expression and function of epithelial cell adhesion molecule EpCAM: where are we after 40 years? Cancer and Metastasis Reviews 39:969-987. DOI: 10.1007/s10555-020-09898-3.

Goddard TD, Huang CC, Ferrin TE. 2007. Visualizing density maps with UCSF Chimera. Journal of Structural Biology 157:281-287. DOI: 10.1016/j.jsb.2006.06.010.

Huang H-P, Chen P-H, Yu C-Y, Chuang C-Y, Stone L, Hsiao W-C, Li C-L, Tsai S-C, Chen K-

PeerJ reviewing PDF | (2021:03:58778:1:1:NEW 22 Apr 2021) 
466

467

468

469

470

471

472

473

474

475

476

477

478

479

480

481

482

483

484

485

486

487

488

489

490

491

492

493

494

495

496

497

498

499

500

501

502

503

504

505

506

507

508

509

510

511

Y, Chen H-F, Ho H-N, Kuo H-C. 2011. Epithelial cell adhesion molecule (EpCAM) complex proteins promote transcription factor-mediated pluripotency reprogramming. The Journal of biological chemistry 286:33520-32. DOI: 10.1074/jbc.M111.256164.

Humphrey W, Dalke A, Schulten K. 1996. VMD: Visual molecular dynamics. Journal of Molecular Graphics 14:33-38. DOI: 10.1016/0263-7855(96)00018-5.

Johannes S, Bercher JD, Blobel CP. 2000. Intracellular maturation and localization of the tumour necrosis factor $\alpha$ convertase (TACE). Biochemical Journal 347:131. DOI: 10.1042/02646021:3470131.

Krissinel E, Henrick K. 2007. Inference of Macromolecular Assemblies from Crystalline State. Journal of Molecular Biology 372:774-797. DOI: 10.1016/j.jmb.2007.05.022.

Liang K-H, Tso H-C, Hung S-H, Kuan I-I, Lai J-K, Ke F-Y, Chuang Y-T, Liu I-J, Wang Y-P, Chen R-H, Wu H-C. 2018. Extracellular domain of EpCAM enhances tumor progression through EGFR signaling in colon cancer cells. Cancer Letters 433:165-175. DOI: 10.1016/j.canlet.2018.06.040.

Lu T-Y, Lu R-M, Liao M-Y, Yu J, Chung C-H, Kao C-F, Wu H-C. 2010. Epithelial cell adhesion molecule regulation is associated with the maintenance of the undifferentiated phenotype of human embryonic stem cells. The Journal of biological chemistry 285:871932. DOI: 10.1074/jbc.M109.077081.

Maetzel D, Denzel S, Mack B, Canis M, Went P, Benk M, Kieu C, Papior P, Baeuerle PA, Munz M, Gires O. 2009. Nuclear signalling by tumour-associated antigen EpCAM. Nature Cell Biology 11:162-171. DOI: 10.1038/ncb1824.

Morris JH, Huang CC, Babbitt PC, Ferrin TE. 2007. structureViz: linking Cytoscape and UCSF Chimera. Bioinformatics 23:2345-2347. DOI: 10.1093/bioinformatics/btm329.

Pan M, Schinke H, Luxenburger E, Kranz G, Shakhtour J, Libl D, Huang Y, Gaber A, Pavšič M, Lenarčič B, Kitz J, Jakob M, Schwenk-Zieger S, Canis M, Hess J, Unger K, Baumeister P, Gires O. 2018. EpCAM ectodomain EpEX is a ligand of EGFR that counteracts EGFmediated epithelial-mesenchymal transition through modulation of phospho-ERK1/2 in head and neck cancers. PLOS Biology 16:e2006624. DOI: 10.1371/journal.pbio.2006624.

Pavšič M, Gunčar G, Djinović-Carugo K, Lenarčič B. 2014. Crystal structure and its bearing towards an understanding of key biological functions of EpCAM. Nature communications 5:4764. DOI: 10.1038/ncomms5764.

Pavšič M, Lenarčič B. 2011. Expression, crystallization and preliminary X-ray characterization of the human epithelial cell-adhesion molecule ectodomain. Acta crystallographica. Section F, Structural biology and crystallization communications 67:1363-6. DOI: 10.1107/S1744309111031897.

Perez MS, Walker LE. 1989. Isolation and characterization of a cDNA encoding the KS1/4 epithelial carcinoma marker. Journal of immunology (Baltimore, Md. : 1950) 142:3662-7.

Pettersen EF, Goddard TD, Huang CC, Couch GS, Greenblatt DM, Meng EC, Ferrin TE. 2004. UCSF Chimera - A visualization system for exploratory research and analysis. Journal of Computational Chemistry 25:1605-1612. DOI: 10.1002/jcc.20084.

Phillips JC, Braun R, Wang W, Gumbart J, Tajkhorshid E, Villa E, Chipot C, Skeel RD, Kalé L, Schulten K. 2005. Scalable molecular dynamics with NAMD. Journal of Computational Chemistry 26:1781-1802. DOI: 10.1002/jcc.20289.

Scheunemann P, Stoecklein NH, Rehders A, Bidde M, Metz S, Peiper M, Eisenberger CF, Schulte Am Esch J, Knoefel WT, Hosch SB. 2008. Occult tumor cells in lymph nodes as a predictor for tumor relapse in pancreatic adenocarcinoma. Langenbeck's archives of surgery

PeerJ reviewing PDF | (2021:03:58778:1:1:NEW 22 Apr 2021) 
512

513

514

515

516

517

518

519

520

521

522

523

524

525

526

527

528

529

530

531

532

533

534

535

536

537

538

539

540

541

542

543

544

545

546

547

548

549

550

551

552

553

554

555

556

557

393:359-65. DOI: 10.1007/s00423-007-0215-0.

Schön MP, Schön M, Mattes MJ, Stein R, Weber L, Alberti S, Klein CE. 1993. Biochemical and immunological characterization of the human carcinoma-associated antigen MH 99/KS 1/4. International journal of cancer. Journal international du cancer 55:988-95.

Shannon P, Markiel A, Ozier O, Baliga NS, Wang JT, Ramage D, Amin N, Schwikowski B, Ideker T. 2003. Cytoscape: a software environment for integrated models of biomolecular interaction networks. Genome research 13:2498-504. DOI: 10.1101/gr.1239303.

Shapovalov M V., Dunbrack RL. 2011. A Smoothed Backbone-Dependent Rotamer Library for Proteins Derived from Adaptive Kernel Density Estimates and Regressions. Structure 19:844-858. DOI: 10.1016/j.str.2011.03.019.

Simon M, Stefan N, Plückthun A, Zangemeister-Wittke U. 2013. Epithelial cell adhesion molecule-targeted drug delivery for cancer therapy. Expert opinion on drug delivery 10:451-68. DOI: 10.1517/17425247.2013.759938.

Spizzo G, Obrist P, Ensinger C, Theurl I, Dünser M, Ramoni A, Gunsilius E, Eibl G, Mikuz G, Gastl G. 2002. Prognostic significance of Ep-CAM AND Her-2/neu overexpression in invasive breast cancer. International Journal of Cancer 98:883-888. DOI: 10.1002/ijc. 10270.

Spizzo G, Went P, Dirnhofer S, Obrist P, Simon R, Spichtin H, Maurer R, Metzger U, Von Castelberg B, Bart R, Stopatschinskaya S, Köchli OR, Haas P, Mross F, Zuber M, Dietrich H, Bischoff S, Mirlacher M, Sauter G, Gastl G. 2004. High Ep-CAM expression is associated with poor prognosis in node-positive breast cancer. Breast Cancer Research and Treatment 86:207-213. DOI: 10.1023/B:BREA.0000036787.59816.01.

Thampoe IJ, Ng JS, Lloyd KO. 1988. Biochemical analysis of a human epithelial surface antigen: differential cell expression and processing. Archives of biochemistry and biophysics 267:342-52.

Trebak M, Begg GE, Chong JM, Kanazireva E V, Herlyn D, Speicher DW. 2001. Oligomeric state of the colon carcinoma-associated glycoprotein GA733-2 (Ep-CAM/EGP40) and its role in GA733-mediated homotypic cell-cell adhesion. The Journal of biological chemistry 276:2299-309. DOI: 10.1074/jbc.M004770200.

Trzpis M, McLaughlin PMJ, de Leij LMFH, Harmsen MC. 2007. Epithelial cell adhesion molecule: more than a carcinoma marker and adhesion molecule. The American journal of pathology 171:386-95. DOI: 10.2353/ajpath.2007.070152.

Tsaktanis T, Kremling H, Pavšič M, von Stackelberg R, Mack B, Fukumori A, Steiner H, Vielmuth F, Spindler V, Huang Z, Jakubowski J, Stoecklein NH, Luxenburger E, Lauber K, Lenarčič B, Gires O. 2015. Cleavage and Cell Adhesion Properties of Human Epithelial Cell Adhesion Molecule (HEPCAM). Journal of Biological Chemistry 290:24574-24591. DOI: $10.1074 /$ jbc.M115.662700.

Varga M, Obrist P, Schneeberger S, Mühlmann G, Felgel-Farnholz C, Fong D, Zitt M, Brunhuber T, Schäfer G, Gastl G, Spizzo G. 2004. Overexpression of epithelial cell adhesion molecule antigen in gallbladder carcinoma is an independent marker for poor survival. Clinical cancer research : an official journal of the American Association for Cancer Research 10:3131-6.

Went PT, Lugli A, Meier S, Bundi M, Mirlacher M, Sauter G, Dirnhofer S. 2004. Frequent EpCam protein expression in human carcinomas. Human Pathology 35:122-128. DOI: 10.1016/j.humpath.2003.08.026.

Yu T, Ma Y, Wang H. 2017. EpCAM Intracellular Domain Promotes Porcine Cell

PeerJ reviewing PDF | (2021:03:58778:1:1:NEW 22 Apr 2021) 
558 Reprogramming by Upregulation of Pluripotent Gene Expression via Beta-catenin $559 \quad$ Signaling. Scientific Reports 7:46315. DOI: 10.1038/srep46315.

560 


\section{Figure 1}

EpEX dimer and the designed mutants.

(A) The structure of EpEX dimer and the designed mutants. The dimer subunits are colored with different shades of grey. The $\beta$-sheet of the CD is depicted in pink and the TYloop is depicted in blue. The sidechains of key interacting residues, as inferred from the crystal structure, are shown as sticks. Residues that were mutated in TYloop ${ }^{\text {mut }}$ and L264C are colored dark blue and orange, respectively. (B) Frequency of inter-subunit inter-residue contacts during EpEX MD simulation. The width of the line between two residues correlates with the frequency of contact formation - a wider line represents more frequent interaction. The size of circles corresponds to the number of interactions with other residues-a larger circle represents more interactions. Residues are colored according to their charge and polarity: positively charged blue, negatively charged red, polar yellow and non-polar grey. The residues of protease-sensitive site Gly79-Arg80-Arg81 are annotated with a star (*).

Residues that were mutated in TYloop ${ }^{\text {mut }}$ are encircled with a red line. (C) Changes in the key inter-subunit inter-residue distances during the first $0.5 \mathrm{~ns}$ of the wild type MD simulation. (D) Changes in the key inter-subunit inter-residue distances during the first $0.5 \mathrm{~ns}$ of the mutant TYloop ${ }^{\text {mut }}$ MD simulation. For distance calculations in $C$ and $D$, equivalent atoms were used for both wild type and mutated residues ( $C_{\mathrm{V}}$ for K/D83, P/D84 and L/D88); for E148 $\mathrm{C}_{6}$ was used, and for W143 and $\mathrm{Y}_{251} \mathrm{C}_{\zeta}$ was used. 
A
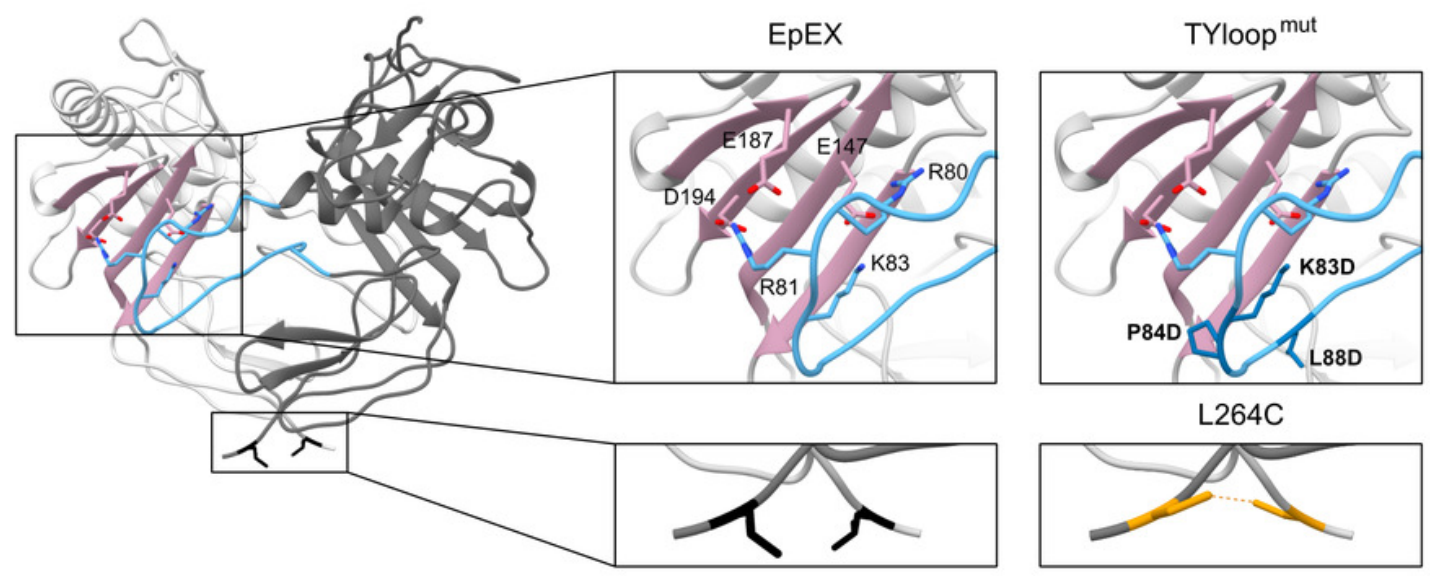

B

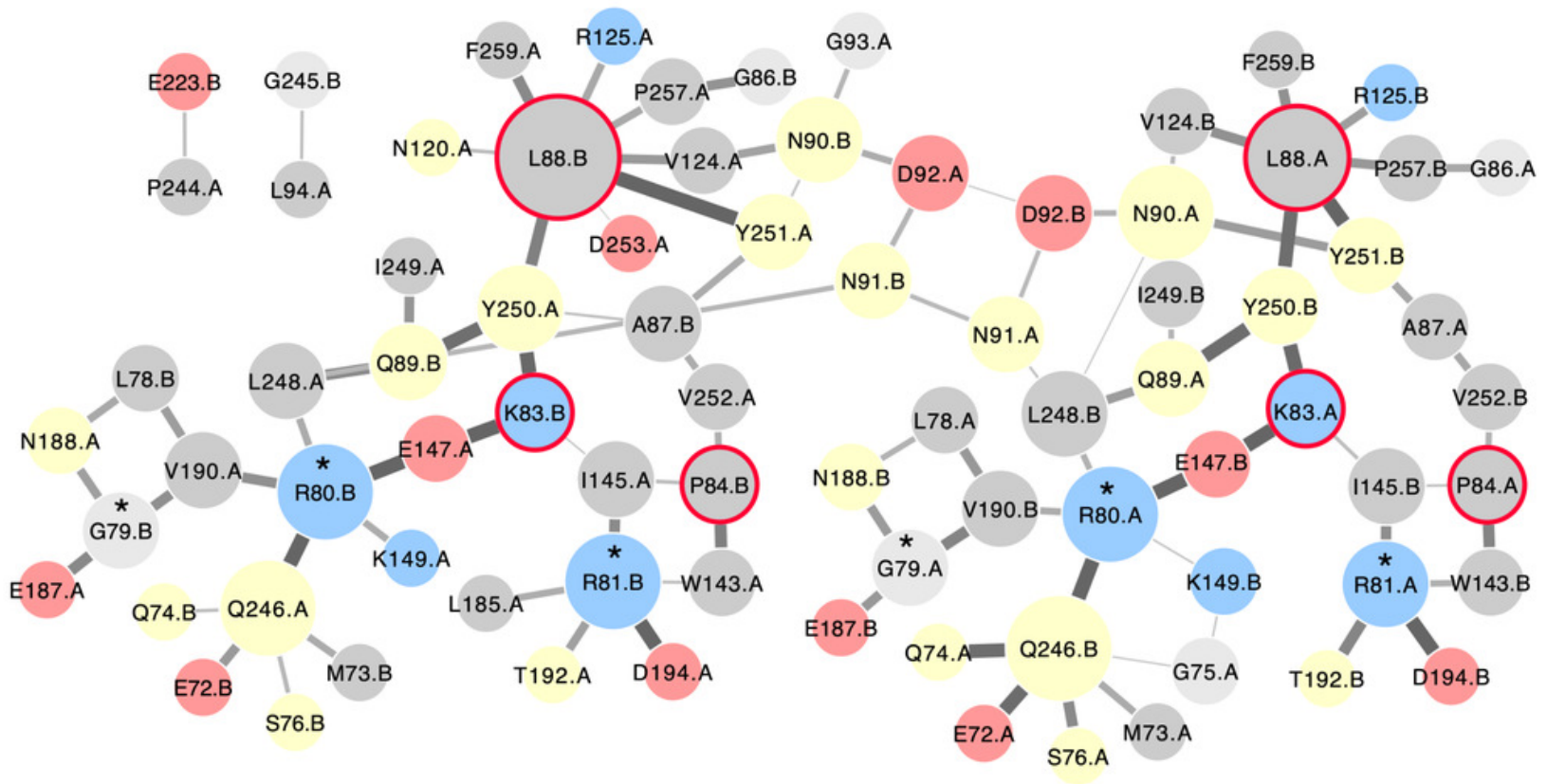

C
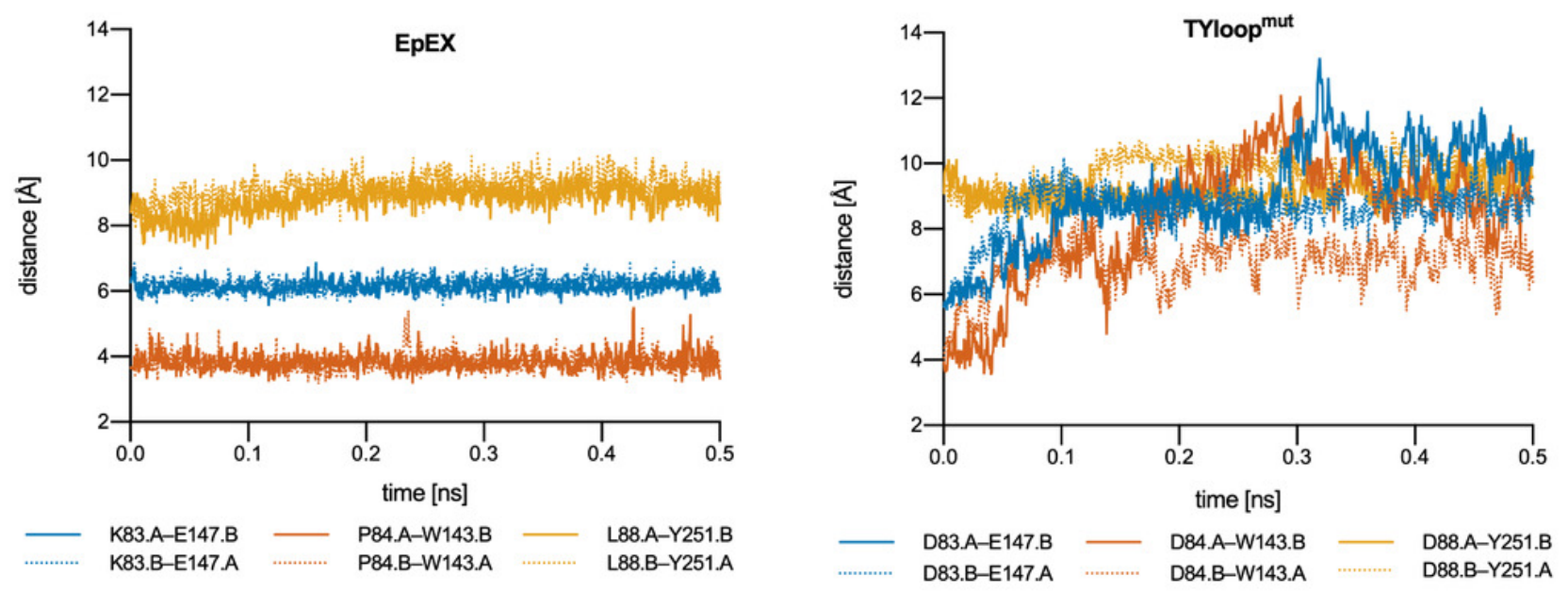
Figure 2

Quality control and oligomeric state analyses of purified EpEX, TYloop ${ }^{\text {mut }}$ and L264C.

(A) Overlay of CD spectra. (B) An overlay of representative SEC elution spectra of purified proteins with the corresponding $M_{w}$ fits. (C) The summary of molecular weights, determined with SEC-RALS/LALS. For each sample, mean values and s.d. from two injections are presented. The predicted mass of a single subunit was calculated from EpEX amino-acid sequence with ProtParam tool [36]. (D) SDS-PAGE analysis under reducing (with DTT) and non-reducing (w/o DTT) conditions.

A

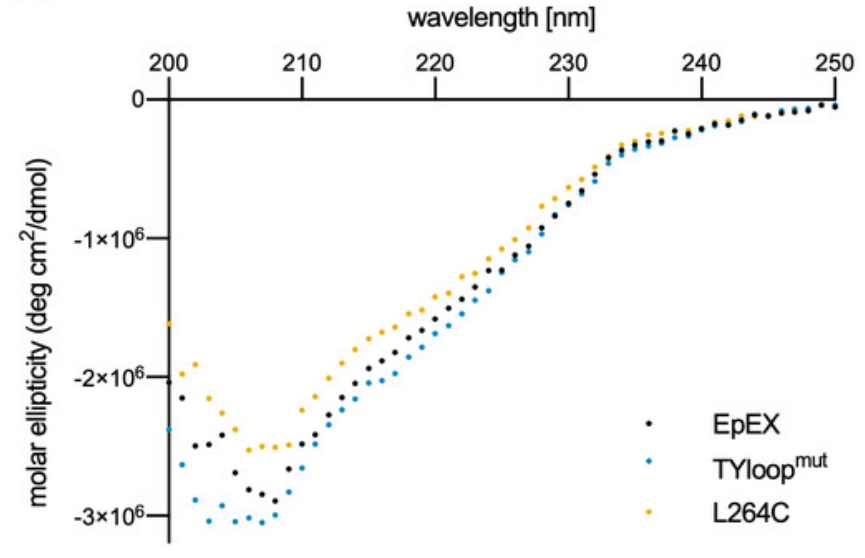

C

\begin{tabular}{cc} 
Sample & MW (kDa) \\
\hline EpEx & $52.8 \pm 0.4$ \\
TYloopmut & $28.7 \pm 0.1$ \\
L264C & $56.0 \pm 0.1$ \\
\hline $\begin{array}{l}\text { predicted mass of } \\
\text { a single subunit }\end{array}$ & 28.5 \\
\hline
\end{tabular}

B

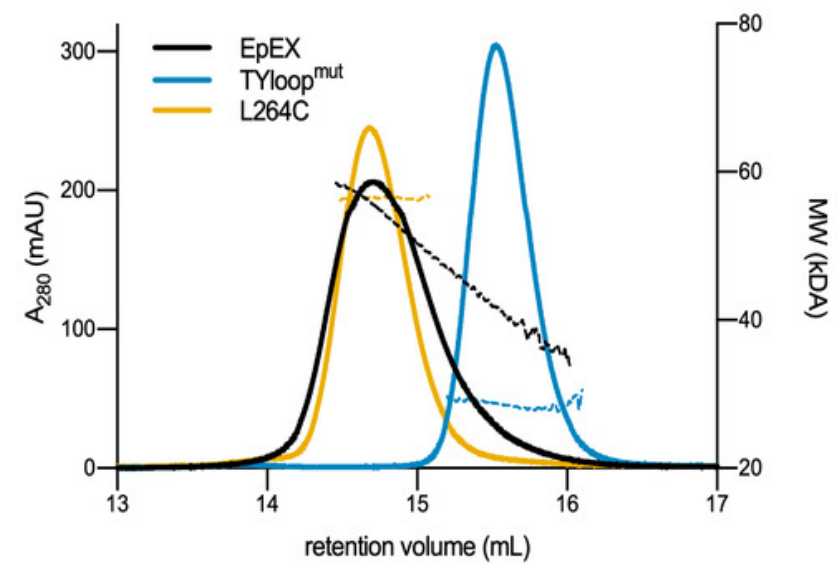

D

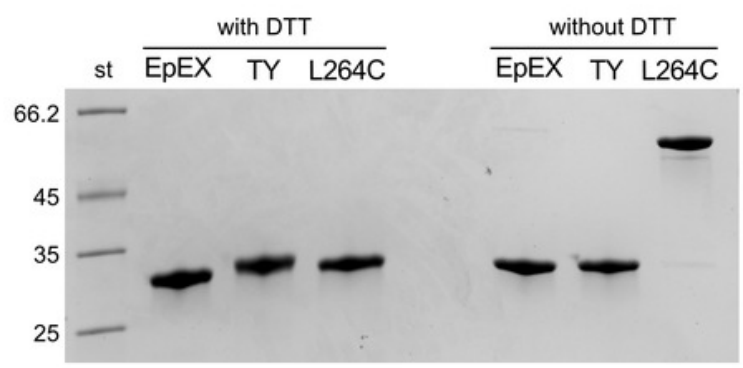




\section{Figure 3}

Cleavage of EpEX and its mutants by TACE-EX

(A) A representative Western blot analysis with anti-histidine tag antibodies. The control samples without the TACE-EX and the control for TACE-EX alone were incubated for $6 \mathrm{~h}$. (B) The comparison of time-dependent reduction of signal intensity. The control sample that was incubated without the TACE-EX is given at $0 \mathrm{~h}$. (C) Histograms representing the reduction of the signal intensity for each analyzed protein. (D) SDS-PAGE analysis of the same representative Western blot analysis. A one-way ANOVA test was performed to identify statistically significant differences between the mean values $(n=3)$ : n.s. non-significant differences, $* * * p<0.0002, * * * * p<0.0001$.

A

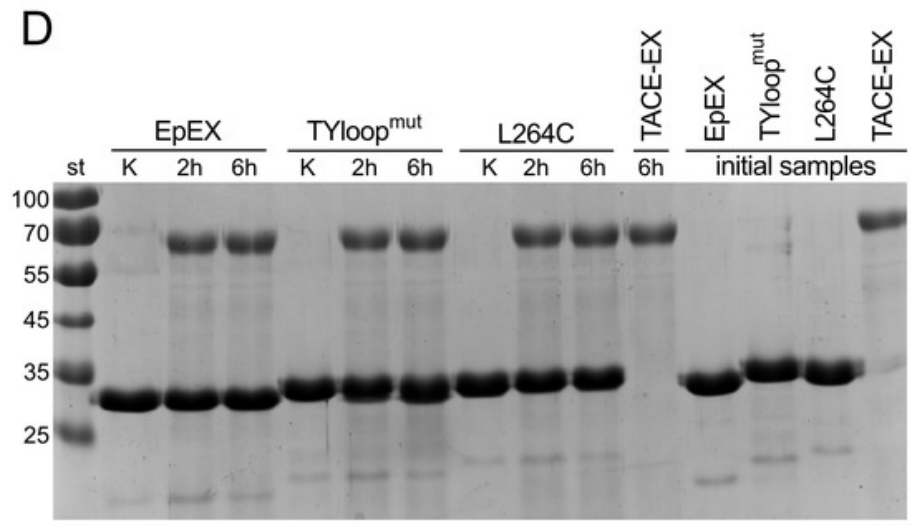

B

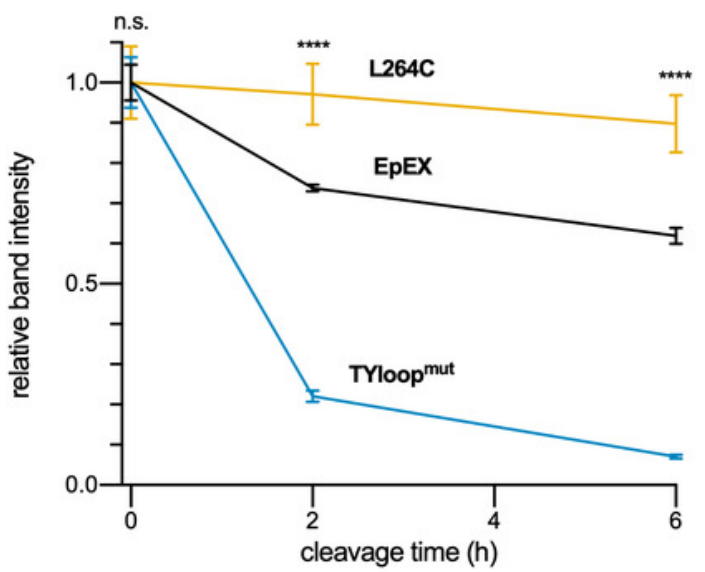

C

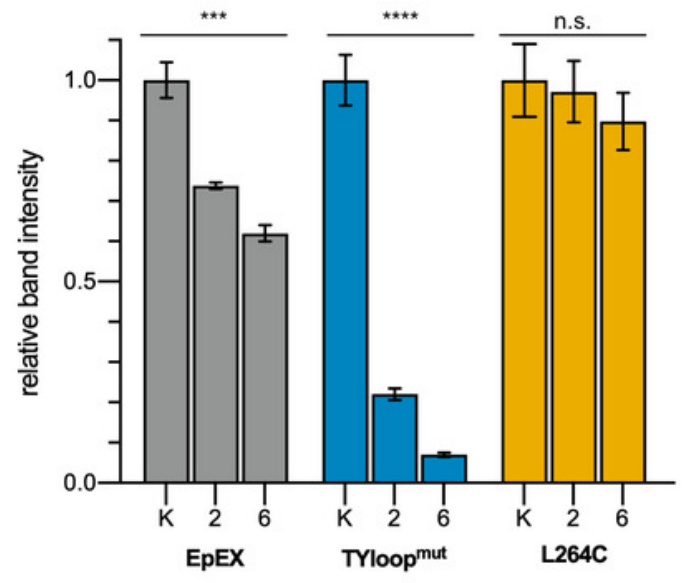




\section{Figure 4}

The proposed model of TACE-mediated EpCAM proteolytic processing, based on EpEX cleavage data.

(A) A Summary of experimental findings. The increased cleavage of the TYloop ${ }^{\text {mut }}$ compared to EpEX is indicated by three arrows. (B) The proposed model of EpCAM cleavage by TACE. Subunits in the EpEX and EpCAM dimers are colored pink and blue, except for the grey transmembrane region. Possible movement of EpEX subunits in the L264C and the destabilized EpCAM dimer is depicted with two positions of the pink subunit and a dashed line with arrows. 
A

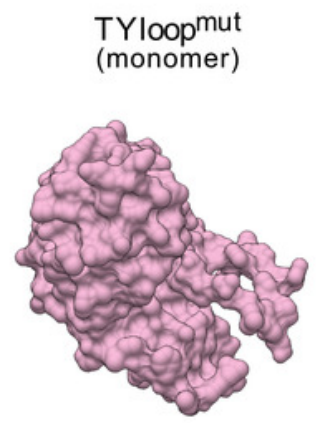

11 cleavage

B

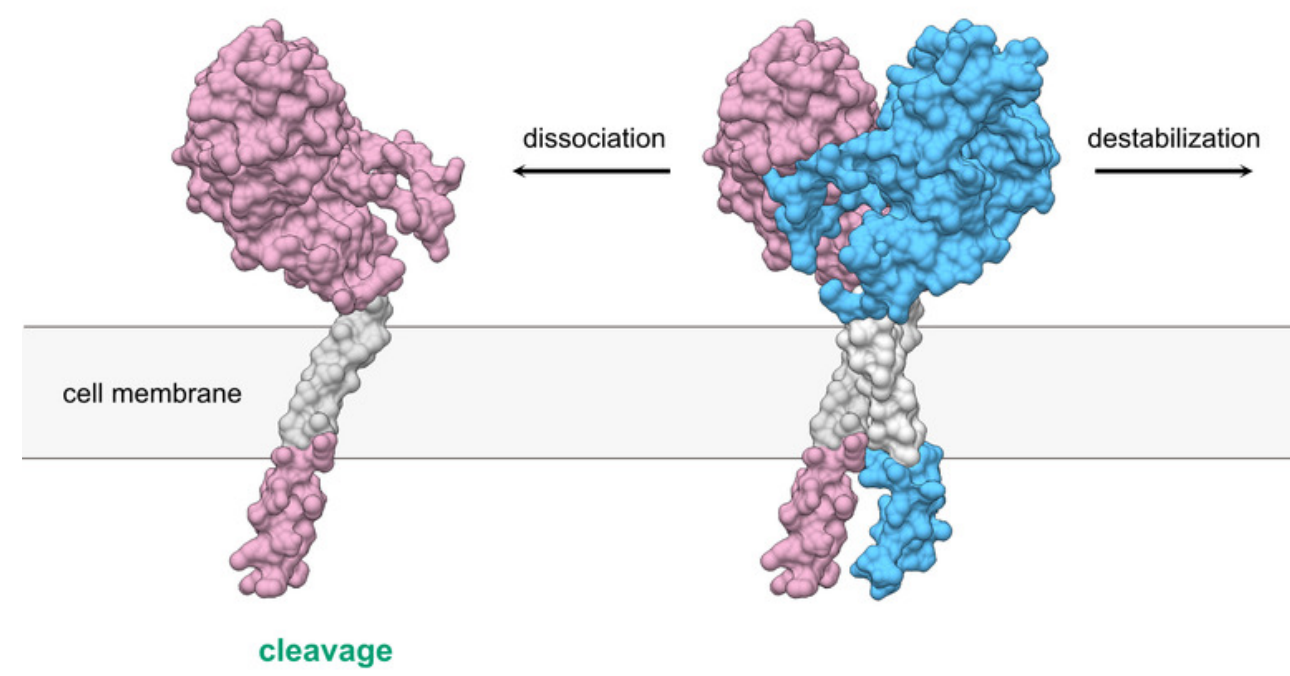

\section{Experimental findings}

EpEX

(non-covalent dimer)

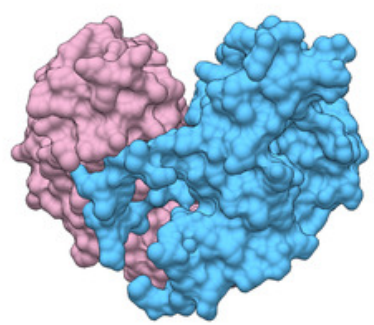

cleavage

\section{Proposed model}

EpCAM dimer

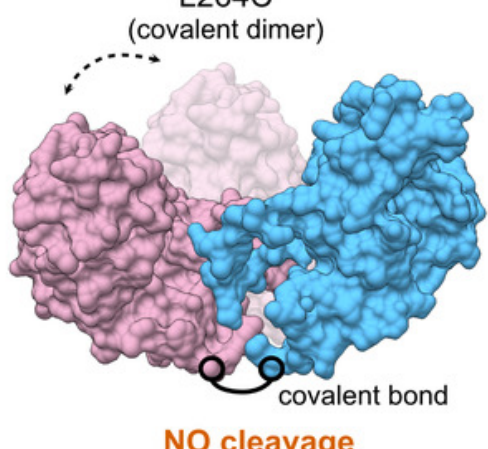

NO cleavage

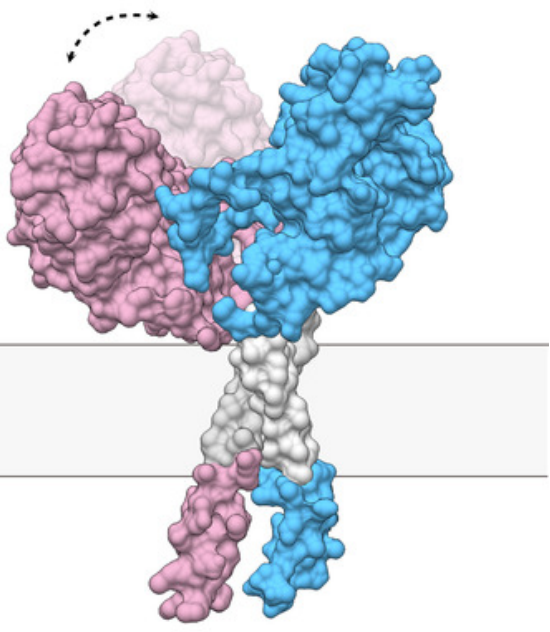

NO cleavage 